\title{
PHOTOGRAPHIC SURFACE PHOTOMETRY OF BRIGHT GALAXIES IN THE M 81 GROUP
}

\author{
R.G. GETOV \\ Rozhen National Astronomical Observatory \\ P.O. Box 136 \\ $B G-4700$ Smolyan \\ Bulgaria
}

\begin{abstract}
A programme for the surface photometry of bright galaxies has been underway at the Rozhen Observatory since 1991. In a series of papers we shall explore the optical morphology, photometric properties and some peculiarities suggestive of galaxy-galaxy interactions of the bright galaxies NGC 2976, NGC 3031, NGC 3034 and NGC 3077 in the M 81 group. Most of the plates were taken with the $2 \mathrm{~m}$ Rozhen Ritchey-Chretien telescope, but at our disposal there are plates from the $6 \mathrm{~m}$ BTA and $2 \mathrm{~m}$ Tautenburg Schmidt telescope. Here we discuss some preliminary results for the optical morphology on the basis of two B-plates from the $2 \mathrm{~m}$ Tautenburg Schmidt telescope. It shows the edge-on like face of NGC 2976, the grand-designed spiral structure of NGC 3031 and the filament-jet peculiarities in NGC 3034 and NGC 3077.
\end{abstract}

\section{Introduction}

The group of the spiral galaxy M 81 (NGC 3031) includes the bright galaxies NGC 2976, NGC 3031, NGC 3034 and NGC 3077 . As a whole the galaxies in the group have been the subject of varied investigation but complete photometrical research has been insufficient. In a series of papers we intend to carry out a detailed surface photometry of these galaxies on the basis of the plates from the $2 \mathrm{~m}$ Tautenburg Schmidt, $2 \mathrm{~m}$ Rozhen Ritchey-Chretien and $6 \mathrm{~m}$ BTA telescopes. We plan to obtain sufficient data with a high resolution for a dependable determination of their optical morphology, photometrical properties, their structure as well as the peculiarities caused by the galaxy-galaxy interactions.

\section{Observational Material and Data Reduction}

In this paper we discuss some preliminary results on the optical morphology of the galaxies on the basis of two B plates from the $2 \mathrm{~m}$ Tautenburg Schmidt telescope, kindly placed at our disposal from Karl Schwarzschild Observatory. They are listed in the table below:

\begin{tabular}{cccccc}
\hline Plate No. & Date & Emulsion & Filter & Band & Exposure \\
\hline \hline 2357 & $06 / 07.02 .1967$ & ZU 2 & GG13 & B & $45^{\min }$ \\
2713 & $01 / 02.03 .1968$ & ZU 2 & GG13 & B & $60^{\min }$ \\
\hline
\end{tabular}


The plates have been measured with a digital microphotometer Joyce Loebl at the Rozhen Observatory. The aperture size used for scanning has a diameter 2.1 arcsec stepped in 1.0 arcsec for NGC 2976 and NGC 3077, a diameter 2.1 arcsec stepped in 1.5 arcsec for NGC 3034 and a diameter 4.1 arcsec stepped in 2.5 arcsec for NGC 3031. The initial images have been scanned with a size of 600 by 600 pixels, i.e. the square areas centred on each galaxy have been scanned with dimension $10.3,15.4$ and 25.7 arcmin respectively.

Data reduction is carried out with the Rozhen Image Processing System (RIPS) on the minicomputer PDP 11/34 at Rozhen. This package (Georgiev 1990) has been specially designed for surface photometry of extended objects.

\section{Data Analysis and Results}

A gradient procedure is applied to the calibrated images. The initial images in 600 by 600 matrix form are reduced twice, then they are smoothed with an aperture 33 by 33 pixels and finally we take out the differences of the initial and smoothed images. In the case of NGC 2976 we observe the twin peaks near its borders. They stand out against the local background with more than 1.3 magnitudes, whereas for the optical nucleus the difference is less than 0.9 magnitude and for the flat disk from 0.2 to 0.5 magnitude. The ten relative bright knots are visible within its border. Hence it follows that NGC 2976 is possibly an edge-on galaxy. The relative low ellipticity is perhaps caused by tidal interactions with the big central galaxy. For NGC 3031 this procedure makes more evident the structural subsystems. The bright kern sticks out among the bulge with more than 3.5 magnitudes, the bulge and the spiral arms stand out against the disk in the range from 0.5 to 2.0 magnitudes and from 0.5 to 1.5 magnitudes respectively. In the case of NGC 3034 it is seen that the bright disk of the galaxy stands out against the local background in the range from 0.5 to 1.0 magnitude whereas for the filaments by the small axis the difference is in the range from 0.2 to 0.5 magnitude. A jet-like structure in the southwest from the nucleus of the NGC 3077 is found. It seems that this small feature about 1 arcmin lies on the elliptical face of the galaxy. For the nuclear region it stands out with more than 2 magnitudes whereas the photometrical nucleus stand out with more than 3 magnitudes.

On the grounds of this analysis we can suppose that the morphological types of the galaxies in the group are affected by the close tidal interaction. A detailed photometric investigation is necessary not only for estimation of the photometrical properties but for a definitive morphological classification also.

\section{References}

Georgiev, Ts., 1990. Astrof. Issl., 30, 111. 